\title{
¿ES NECESARIO PROFESIONALIZAR LA FIGURA DEL TUTOR DE ESPECIALISTAS EN FORMACIÓN SANITARIA?
}

\section{IS IT NECESSARY TO PROFESSIONALIZE THE FIGURE OF THE ACADEMIC ADVISOR OF SPECIALISTS IN SANITARY TRAINING}

\author{
Miriam Martínez Juárez, Pilar Martínez Clares, Eduardo B. Romero Sánchez \\ Universidad de Murcia, España \\ Jesús Miguel Muñoz Cantero \\ Universidade da Coruña, España
}

\begin{abstract}
Resumen: El sistema de formación de especialistas sanitarios en nuestro país es suficientemente maduro, pero se ha de enfrentar a nuevos retos, como es el caso de la profesionalización del tutor de especialistas en formación. La actual normativa que regula la formación especializada atribuye importancia a esta figura pero apenas la perfila. La gran transformación estructural, replantea la necesidad de concebir nuevos profesionales a los que se les pueda atribuir competencias no requeridas hasta el momento. Para ayudar a la adaptación de todos estos cambios, es preciso nuevos sistemas de enseñanza-aprendizaje basados en competencias que faciliten un aprendizaje a lo largo de la vida, para contribuir al nacimiento del profesional requerido y demandado en el siglo XXI. Somos conscientes que ni las organizaciones sanitarias ni el perfil del profesional de la salud han quedado al margen de las nuevas exigencias del entorno, por lo que nos preguntamos si el tutor de especialistas en formación está preparado para asumir estas responsabilidades, siendo necesario y prioritario su profesionalización.
\end{abstract}

Palabras clave: función tutorial, tutor de especialistas en formación (sanitario), profesionalidad, perfil profesional.

\begin{abstract}
The training system of health care professionals in our country is mature enough, but it has to face new challenges, as it is the case of the professionalization of the academic advisor of specialists in formation. The current regulation governing the specialized training, attaches importance to this figure but scarcely portrays it. The large structural transformation raises the need to conceive new professionals to which to attribute competencies that have not been required to date. To assist the adaptation to all these changes, we need new teaching-learning systems based on competencies to facilitate learning throughout life, to contribute to birth of the kind of professional required and demanded in the XXI century. We are aware that neither the health care organizations, nor the profile of health care professionals have remained on the sidelines of the new requirements of the environment. Therefore, we wonder if the academic advisor of specialists in training is prepared to assume these responsibilities, being necessary and priority, its professionalization.
\end{abstract}

Key Words: tutorial guidance, tutor of specialists in formation, training (sanitary), professionalism, professional profile.

\section{INTRODUCCIÓN}

El sistema MIR (ver cuadro adjunto) en nuestro país tiene un largo desarrollo histórico, se encuentra suficientemente acreditado y su prestigio está internacionalmente elogiado. Son varias las razones que explican esta alta consideración: arraigo, amplia regulación normativa, universalidad, ecuanimidad para la obtención de plazas, acreditación docente de las Unidades que la imparten, evaluación continuada mediante sistemas de tutorías y Comisiones de Docencia y escasez de vías alternativas de formación entre otras muchas.

Tradicionalmente se habla de MIR (Médico Interno y Residente) porque en el modelo anglosajón los médicos que se iniciaban en los departamentos como ayudantes se denominaban así porque vivían los primeros años en el hospital. En el Estado Español sólo se va a incorporar esta denominación y se va a adaptar el espíritu de esta figura profesionalizadora del médico a las características de nuestro sistema de formación médica especializada. Hoy en día resulta más correcto referirse a los MEF (Médico Especialista en Formación), pero las siglas MIR siguen estando tan arraigadas que es difícil hacer uso de la nueva terminología aún cuando resulta más correcta y adecuada.

Correspondencia: Jesús Miguel Muñoz Cantero. Área de Métodos de Investigación y Diagnóstico en Educación, Universidad de la Coruña, España. Correo Electrónico: munoz@udc.es 
Sin embargo, y precisamente para seguir manteniendo el reconocimiento alcanzado y estos buenos resultados, estamos obligados a un análisis constante del propio sistema, para que se adapte a la realidad social y profesional actual.

En la literatura se ha tratado con empeño el perfil que debe tener un residente para llegar a ser un buen profesional, pero no se ha profundizado tanto en el que debe tener un tutor. Esta falta de nitidez en la definición de sus funciones, de sus competencias y en los límites de su actuación hace que no se avance, como debiera, su proceso de profesionalización.

Lo que ya nadie cuestiona, es la enorme importancia de la función tutorial y de la figura del tutor dentro de este sistema formativo. $Y$ esto es así, porque la relación residente-tutor constituye el eje fundamental sobre el que gira todo el proceso formativo, en la medida en que el sistema de residencia implica una formación teórica y práctica, programada y tutelada, con una participación personal y progresiva.

El Tutor es el especialista encargado de la planificación, supervisión y evaluación de todo el proceso formativo del residente. En nuestro país, con el R.D.183/2008, publicado en el BOE de 21 de Febrero de 2008, se plantea por primera vez una normativa con un reconocimiento de esta figura. Es competencia de las Comunidades Autónomas definir el procedimiento de nombramiento de los Tutores, así como su reconocimiento. Este reconocimiento lleva implícito la acreditación y evaluación de los mismos.

Cuando cualquier profesional de la Salud, un médico, enfermero, psicólogo residente $u$ otro se integra en el sistema asistencial es preciso garantizar su formación con tendencia a la excelencia. Este hecho implica disponer de dos instrumentos: por una parte, de un plan de formación individualizado, de acuerdo a las competencias a alcanzar establecidas en el Programa Formativo de la Especialidad correspondiente, y, por otra parte, de un adecuado sistema de supervisión de ese plan de formación (Morán, 2003). Un especialista sanitario del siglo XXI debe ser no sólo un experto en su especialidad clínica, sino también un consejero de la salud, un gestor de recursos, poseer habilidades de comunicación con pacientes y familiares, saber trabajar en equipo, ser un gestor del conocimiento y albergar valores profesionales referidos a conocimientos y conductas acordes a unos principios éticos y morales de la sociedad (Canadian Medical Education Directions for Specialist 2000 Project [CanMEDS 2000 Project], 1996). Estas competencias no parece que puedan ser alcanzadas por el residente con una simple integración funcional en la actividad asistencial del centro y dejándose llevar por las rutinas de ese centro o servicio.

Actualmente en nuestro país, el sistema de formación especialistas sanitarios es suficientemente maduro, pero se ha de enfrentar a nuevos retos, como es el caso de la profesionalización del tutor de especialistas en formación. Desde nuestra formación pedagógica, creemos que para la formación integral de un residente es necesario establecer un programa formativo adaptado al centro, evaluable y flexible en función del aprendizaje del mismo, junto a un responsable en el servicio que supervise y garantice el cumplimiento de ese programa, haciendo compatible la realidad formativa con la realidad asistencial. La responsabilidad de la gestión de un servicio o unidad recae en la jefatura del mismo y, al igual que se realiza un reparto de tareas organizativas y asistenciales entre los mandos intermedios de dicho servicio o unidad, la formación especializada debe de tener, en la lógica de la organización del trabajo, un responsable (Fernández- Ríos, 1995).

La figura del tutor de residentes es una necesidad organizativa básica y no simplemente el cumplimiento de un formalismo. Un servicio que dispone de un tutor que realmente programa, coordina, dirige, supervisa y evalúa la progresión de un residente, es un servicio que ha asumido con todas sus consecuencias las responsabilidades de estar acreditado para la formación especializada y puede demostrar a la sociedad la calidad de los especialistas que forma.

La necesidad del tutor de especialistas en formación es algo que ya nadie se cuestiona. Por ello, estamos de acuerdo en que es preciso avanzar. La cuestión que nos planteamos a continuación es cómo podemos hacerlo.

Una buena manera sería contribuyendo a profesionalizar la figura del tutor de residentes, figura que se inserta en una sociedad en constante evolución y que exige, cada vez con mayor vehemencia, cambios en los comportamientos y actitudes de las personas que se integran en ella. 


\section{LA TRANSFORMACIÓN ESTRUCTURAL}

La sociedad de nuestros días se caracteriza principalmente por el saber, donde el conocimiento es esencial para el desarrollo de cualquier actividad socio- laboral. Por ello, a la sociedad nacida en los últimos años del siglo XX se le ha denominado "Sociedad de la Información y del Conocimiento", dados los cambios, evoluciones o revoluciones acaecidas en ciertos ámbitos.

Los cambios en los que nos vemos inmersos hoy en día provocan la gran transformación estructural actual, que induce cambios en las lógicas de producción y en las propias organizaciones y a su vez estimulan cambios en los sistemas de enseñanza/aprendizaje, facilitando un aprendizaje a lo largo de la vida. Esta transformación estructural, queda representada gráficamente por Echeverría (2001-2008) en el "Reloj de arenas movedizas" (gráfico 1).

Respecto a la transformación tecnológica constatamos como la innovación tecnológica se ha convertido en una constante hoy en día.
Las Tecnologías de la Información y la Comunicación (TICs) se encuentran presentes en casi todas las parcelas de nuestra vida, influyendo y condicionando nuestra manera de relacionarnos con los demás, de trabajar, de disfrutar del ocio y tiempo libre... La generalización del uso de las TICs se ha implantado en una amplia gama de facetas de la actividad humana, configurando la cultura del digitalismo (Echeverría, 2001). No obstante las TICs deben evitar que la brecha digital genere capas de marginación como resultado de la analfabetización digital.

Igualmente, respecto a la transformación económica, nos vemos inmersos en un proceso de globalización caracterizado por la transformación del tiempo y el espacio, en el que desaparecen las distancias y el tiempo es percibido como un continuo. Algunas muestras de la tendencia globalizadora de nuestros días son la libre circulación de capitales dentro de la Unión Europea, los criterios de convergencia del euro, las fusiones mundiales de empresas, los movimientos de las bolsas...

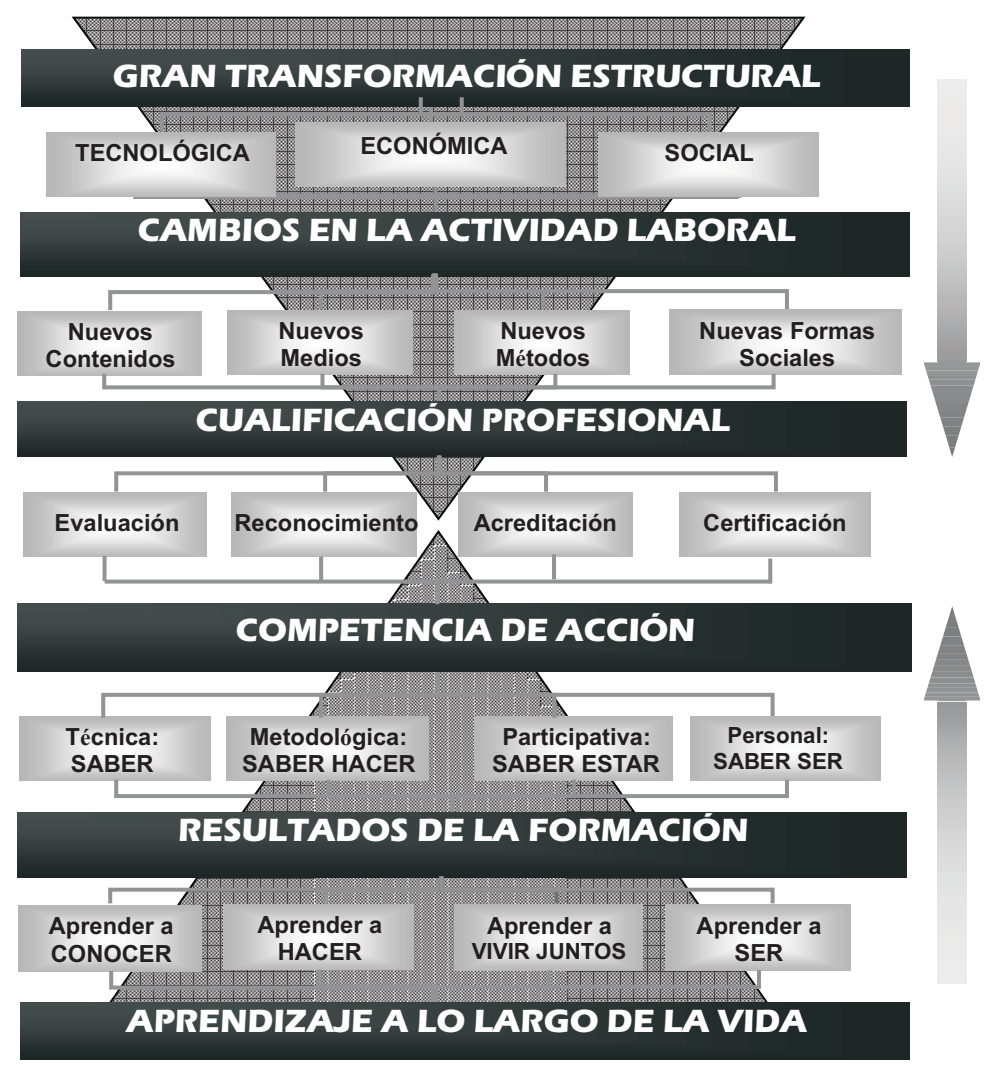

Gráfico 1. Reloj de arenas movedizas (Echeverría, 2008: 27) 
Estas son características de la transformación económica del siglo XXI mediatizada por la globalización, fenómeno real que posibilita reconquistar un importante poder negociador.

Finalmente, respecto a la transformación social, vemos que surge de la sinergia de las dos anteriores. Como características de este cambio podemos observar como las diferencias entre países desarrollados y subdesarrollados son cada vez mayores; a su vez, dentro de los países desarrollados se hace también más patente el desajuste entre ricos y pobres; al mismo tiempo, la población mundial se multiplica de forma desigual, y nos encontramos, además, con el fenómeno del desempleo, que afecta particularmente a jóvenes, mujeres y parados de larga duración, pero que a corto plazo puede afectar también a personas con escasa o nula cualificación, cuyos puestos comienzan a pasar progresivamente a manos de inmigrantes.

Efecto derivado de estas grandes transformaciones son algunos de los más notables cambios producidos en las lógicas de producción y en las organizaciones sociolaborales, así como en sus formas sociales de desarrollo.

Es evidente que la organización del trabajo ha evolucionado mucho, sobre todo desde el primer cuarto del siglo $\mathrm{XX}$, es decir, desde el surgimiento de una nueva perspectiva organizacional denominada por Taylor (1911) como "organización científica del trabajo".

Actualmente, el trabajo manual es sustituido por máquinas y controles automáticos, liberando de muchas horas al personal en los procesos de producción, control y distribución, particularmente en aquellos que implican un mayor esfuerzo. Con la robotización y los controles informatizados, los trabajadores se liberan de la ejecución de muchas tareas y de la manipulación de las máquinas para pasar a tareas más sofisticadas y complejas, como son el diseño y la simulación (Fernández- Ríos, 1995). Por su parte, el trabajo administrativo también ha experimentado cambios de magnitudes impensables sobre todo con la introducción y el uso de las TICs.

Los cambios mencionados han dado lugar a nuevos contenidos y medios de trabajo, que junto al uso combinado de las TICs, comienzan a repercutir poderosamente en la organización del mismo. La automatización, el trabajo a distancia, los horarios flexibles y libres... ofrecen a los empleados nuevas e insospechadas posibilidades (Fernández- Ríos, 1995), así como nuevas formas sociales de trabajo.

Estas nuevas formas sociales de trabajo vienen propiciadas por nuevos métodos de trabajo. La organización jerárquica tradicional, con su pirámide de poder bien dibujada y perfilada, tiende a extinguirse y emerge en su lugar estructuras laborales más flexibles. Esta nueva estructura de las organizaciones, caracterizada por la flexibilidad, se convierte en una necesidad para ellas, dada la competitividad del mercado actual: "Contra la fluctuación del mercado, se adopta la flexibilización en la elaboración de bienes y servicios. Contra el dilema de los stocks, se incrementa la flexibilidad de la organización del trabajo (...). Contra la incertidumbre de los costes de trabajo, se baraja la posibilidad de utilizar éste en mayor o menor medida" (Echeverría, 2008: 39). Este hecho da lugar a menos puestos de trabajo estable y a otras formas sociales de trabajo como el autoempleo, el teletrabajo, contratos temporales, trabajo a tiempo parcial, subcontratación,...

Esta gran transformación estructural, que vive la sociedad de hoy en día, no sólo incide en propiciar cambios en las lógicas de producción y en las organizaciones, sino que también exige cambios en los sistemas de enseñanza/ aprendizaje. Se ha producido una evolución en los requerimientos profesionales hasta llegar a la actual demanda de competencias profesionales, facilitando y favoreciendo un aprendizaje a lo largo de la vida.

Pero está claro que sólo se podrán desarroIlar y adquirir las competencias profesionales si los sistemas formativos cambian o evolucionan en paralelo a la transformación estructural, a la que estamos haciendo referencia, es decir, si hay un cambio de enfoque de una enseñanza tradicional a una formación basada en competencias, que se apoye en los cuatro pilares básicos que anunció Delors en su informe "La educación encierra un tesoro"; nos referimos a aprender a conocer, aprender a hacer, aprender a vivir juntos y aprender a ser.

Este autor defiende que "mientras los sistemas educativos formales propenden a dar prioridad a la adquisición de conocimientos, en detrimento de otras formas de aprendizaje, importa concebir la educación como un todo" (Delors, 1996:17).

Constatamos como estos cuatro pilares de la educación se pueden relacionar con los 
cuatro componentes de la Competencia de Acción Profesional (cuadro1); nos referimos a la competencia técnica, la competencia metodológica, la competencia participativa y la competencia personal.

La Competencia de Acción Profesional engloba este conjunto de conocimientos, habilidades y actitudes aprendidos a raíz del cambio producido tanto en los objetivos como en los resultados del proceso de formación o de enseñanza/aprendizaje.

Estamos de acuerdo con Echeverría (2005:17) cuando expresa que "esta transformación en los requerimientos profesionales exige un mayor acercamiento entre los sistemas formativos y laborales y una mejor coordinación entre ofertas y exigencias, para ofrecer respuestas válidas y actualizadas a las necesidades, que permitan el desarrollo permanente de las personas". Por tanto, es preciso que los cambios producidos en las lógicas de producción y en las organizaciones confluyan con los que deben producirse irremediablemente en el contexto formativo y educativo, buscando el equilibrio en el mercado laboral. En este equilibrio, las cualificaciones personales, adquiridas a partir de las cualificaciones estructurales establecidas por el sistema educativo, deben corresponderse con las cualificaciones institucionales demandadas desde las organizaciones.

\begin{tabular}{|c|c|}
\hline $\begin{array}{l}\text { PILARES DE LA EDUCACIÓN } \\
\text { (DELORS, 1996) }\end{array}$ & $\begin{array}{l}\text { COMPETENCIA DE ACCIÓN } \\
\text { PROFESIONAL } \\
\text { (ECHEVERRÍA, 2001-2008) }\end{array}$ \\
\hline $\begin{array}{c}\text { APRENDER A CONOCER } \\
\text { Combinar el conocimiento de la cultura } \\
\text { general con la posibilidad de profundizar en } \\
\text { niveles más específicos. }\end{array}$ & $\begin{array}{l}\text { COMPETENCIA TÉCNICA (SABER) } \\
\text { Dominio integrado de conocimientos } \\
\text { teóricos y prácticos, incluyendo el } \\
\text { conjunto de saberes específicos y la } \\
\text { gestión de esos conocimientos }\end{array}$ \\
\hline $\begin{array}{c}\text { APRENDER A HACER } \\
\text { Capacitación para hacer frente a diversas } \\
\text { situaciones y experiencias vitales y } \\
\text { profesionales }\end{array}$ & $\begin{array}{l}\text { COMPETENCIA METODOLÓGICA } \\
\text { (SABER HACER) } \\
\text { Habilidades, destrezas y hábitos fruto } \\
\text { del aprendizaje y de la experiencia que } \\
\text { garantizan la calidad productiva }\end{array}$ \\
\hline $\begin{array}{c}\text { APRENDER A CONVIVIR } \\
\text { Dirigido a la comprensión, } \\
\text { interdependencia y resolución de conflictos }\end{array}$ & $\begin{array}{l}\text { COMPETENCIA PARTICIPATIVA } \\
\text { (SABER ESTAR) } \\
\text { Dominio de la cultura del trabajo, del } \\
\text { ámbito social y la participación en el } \\
\text { entorno }\end{array}$ \\
\hline $\begin{array}{l}\text { APRENDER A SER } \\
\text { Desarrollo de la autonomía, juicio, } \\
\text { responsabilidad y desarrollo de sus } \\
\text { posibilidades }\end{array}$ & $\begin{array}{l}\text { COMPETENCIA PERSONAL (SABER } \\
\text { SER) } \\
\text { Valores, comportamientos y actitudes, } \\
\text { poseer una imagen realista de sí mismo } \\
\text { y actuar conforme a esto }\end{array}$ \\
\hline
\end{tabular}




\section{HACIA LA PROFESIONALIZACIÓN DEL TUTOR DE ESPECIALISTAS EN FORMACIÓN}

No obstante, a tenor de lo expuesto anteriormente, no podemos olvidar que las demandas profesionales han evolucionado notablemente en los últimos tiempos, tal y como se señalan en el cuadro 2 que Delcourt (1991) ofrece, y en el que sintéticamente mostramos la evolución de los requerimientos profesionales.

Para Vargas y cols. (2001), "las nuevas necesidades que el ambiente competitivo ha impuesto a las organizaciones, requieren de respuestas más rápidas que no se pueden dar bajo las tradicionales formas de organización del trabajo. Una rápida adaptación al cambio, aceptar desafíos, cambiar y aprender continuamente, son imperativos para cualquier empresa (organización, institución) en un mundo globalizado.
Lograr estas características implica que la empresa también se convierta en un "equipo competente" conformado, obviamente, por trabajadores competentes".

La actual transformación estructural se caracteriza fundamentalmente por el cambio generado en el escenario socio-laboral y por ende en sus requerimientos (Echeverría, 2001-2008):

- En los años 60' - 70' se pedían esencialmente capacidades para realizar actividades definidas y vinculadas a una determinada profesión. Primaba la noción de tarea, sobre la de función integrada en el sistema de empresa.

- A partir de la década de los 80', comenzó a demandarse cualificaciones que incluyesen conocimientos y destrezas para ejercer una amplia gama de actividades laborales.

\section{EVOLUCIÓN DE LOS REQURIMIENTOS PROFESIONALES}

\begin{tabular}{|c|c|}
\hline $\begin{array}{l}\text { Trabajo según especificaciones y } \\
\text { según órdenes. }\end{array}$ & $\begin{array}{l}\text { Autonomía, iniciativa, responsabilidad } \\
\text { y creatividad esperadas. }\end{array}$ \\
\hline Trabajo fragmentado, especializado. & $\begin{array}{l}\text { Trabajo enriquecido horizontal y } \\
\text { verticalmente. }\end{array}$ \\
\hline $\begin{array}{l}\text { Trabajo de base energética (fuerza } \\
\text { física ejercida sobre materiales u } \\
\text { objetos). }\end{array}$ & $\begin{array}{l}\text { Trabajo de base informática (trabajo } \\
\text { intelectual y transmisión de } \\
\text { informaciones o de signos). }\end{array}$ \\
\hline $\begin{array}{l}\text { Relación física con el producto o el } \\
\text { material. }\end{array}$ & $\begin{array}{l}\text { Relación mediatizada con el producto o } \\
\text { el material. }\end{array}$ \\
\hline $\begin{array}{l}\text { Habilidad, destreza y velocidad de } \\
\text { ejecución en el plano manual. }\end{array}$ & $\begin{array}{l}\text { Velocidad de percepción, de reacción, } \\
\text { de coordinación en el plano intelectual. }\end{array}$ \\
\hline $\begin{array}{l}\text { Separación entre el pensamiento y el } \\
\text { gesto. }\end{array}$ & $\begin{array}{l}\text { Entrelazamiento del pensamiento y del } \\
\text { gesto (resolución de problemas). }\end{array}$ \\
\hline $\begin{array}{l}\text { Trabajo pesado, en ocasiones peligroso } \\
\text { e insaludable. }\end{array}$ & $\begin{array}{l}\text { Predominio del trabajo individual y de } \\
\text { situaciones de estrés. }\end{array}$ \\
\hline Ajuste mecánico de las personas. & $\begin{array}{l}\text { Ajustes en función de las exigencias de } \\
\text { las situaciones y de las relaciones. }\end{array}$ \\
\hline $\begin{array}{l}\text { Gestión de situaciones rutinarias o } \\
\text { previstas. }\end{array}$ & $\begin{array}{l}\text { Gestión caso por caso sin demasiadas } \\
\text { rutinas. }\end{array}$ \\
\hline $\begin{array}{l}\text { Predominio de trabajadores manuales, } \\
\text { especializados y cualificados. }\end{array}$ & $\begin{array}{l}\text { Predominio de trabajadores } \\
\text { cualificados, de técnicos, de ingenieros } \\
\text { y de directivos. }\end{array}$ \\
\hline $\begin{array}{l}\text { Horarios y calendarios de trabajos } \\
\text { fijos. }\end{array}$ & $\begin{array}{l}\text { Autonomía y flexibilidad del horario } \\
\text { de trabajo y del calendario. }\end{array}$ \\
\hline Homogeneidad de las cualificaciones. & $\begin{array}{l}\text { Gama diversificada de competencias } \\
\text { en los trabajadores. }\end{array}$ \\
\hline $\begin{array}{l}\text { Clasificaciones profesionales rígidas } \\
\text { en función de las competencias y } \\
\text { experiencias. }\end{array}$ & $\begin{array}{l}\text { Clasificaciones en función de las } \\
\text { capacidades de asimilación de nuevos } \\
\text { conocimientos y de la adaptabilidad. }\end{array}$ \\
\hline Cualificaciones intercambiables. & $\begin{array}{l}\text { Cualificaciones heterogéneas y } \\
\text { clasificaciones idiosincrásicas. }\end{array}$ \\
\hline $\begin{array}{l}\text { Remuneración en función del } \\
\text { rendimiento y de la productividad. }\end{array}$ & $\begin{array}{l}\text { Remuneración en función de los riegos } \\
\text { y de los problemas que deben } \\
\text { resolverse, así como de los objetivos } \\
\text { alcanzados. }\end{array}$ \\
\hline Escasa autorrealización. & $\begin{array}{l}\text { Inversión personal e importancia de la } \\
\text { autorrealización. }\end{array}$ \\
\hline Sindicatos de sectores y de clase. & Sindicatos profesionales y de empresa. \\
\hline
\end{tabular}

Cuadro 2. Evolución de los requerimientos profesionales (Delcourt, 1991) 
- Desde el inicio de la década de los 90’ hasta la actualidad, esta intersección de cualificaciones se ha impuesto un profundo replanteamiento de los perfiles profesionales, que de forma progresiva tienden a definirse en términos de competencias, entendidas como un fenómeno complejo desde la acción.

Actualmente, la simple idea que un profesional competente, es aquel que posee los conocimientos y habilidades que le posibilitan desempeñarse con éxito en una profesión específica ha quedado atrás. Sustituida por la comprensión de la competencia profesional como fenómeno complejo, que expresa las potencialidades de la persona para orientar su actuación en el ejercicio de la profesión con iniciativa, flexibilidad y autonomía, en escenarios heterogéneos y diversos, a partir de la integración de conocimientos, habilidades, motivos y valores que se expresan en un desempeño profesional eficiente, ético y de compromiso social.

En palabras de la UNESCO (Delors, 1996), no basta conocer y saber hacer, es necesario ser profesional. De esta forma podemos afirmar que es competente "quien dispone de los conocimientos, destrezas y aptitudes para ejercer una profesión, puede resolver los problemas profesionales de forma autónoma y flexible, y está capacitado para colaborar en su entorno profesional y en la organización del trabajo" (Bunk, 1994:9).
Por lo tanto, "hoy en día, las capacidades estáticas asociadas a cada profesión, así como los modelos y las técnicas de gestión tradicionales, resultan poco flexibles e inadecuados a los lugares de trabajo que exigen de las personas trabajadoras y de sus mandos justo lo contrario" (Echeverría, 2003:7). Estos nuevos requerimientos que el ambiente competitivo ha impuesto a las organizaciones precisan un equilibrio entre el puesto de trabajo y el profesional por lo que "cada vez más la profesionalidad se configura dentro de la relación dialéctica entre el puesto de trabajo y la organización donde se ejerce", tal y como represnta Alex (1991) en el gráfico 2, citado en Echeverría (2001-2008).

Si nos situamos en el contexto que nos interesa en este trabajo, es decir, el sanitario, vemos como la figura del tutor de especialistas en formación se inserta en este entorno al que nos hemos referido anteriormente. El tutor sanitario es un profesional al que, desde su centro de trabajo, se le exige realizar una serie de funciones y competencias ante las que no sabe cómo actuar o comportarse, es decir, para las que no es competente. En su caso, no existe equilibrio entre lo que le demanda la organización y lo que puede ofrecer.

Para contribuir entonces a su profesionalización es preciso determinar qué competencias es necesario que adquiera, a partir de una serie de funciones prefijadas, y procurar con ello un cambio en su formación.

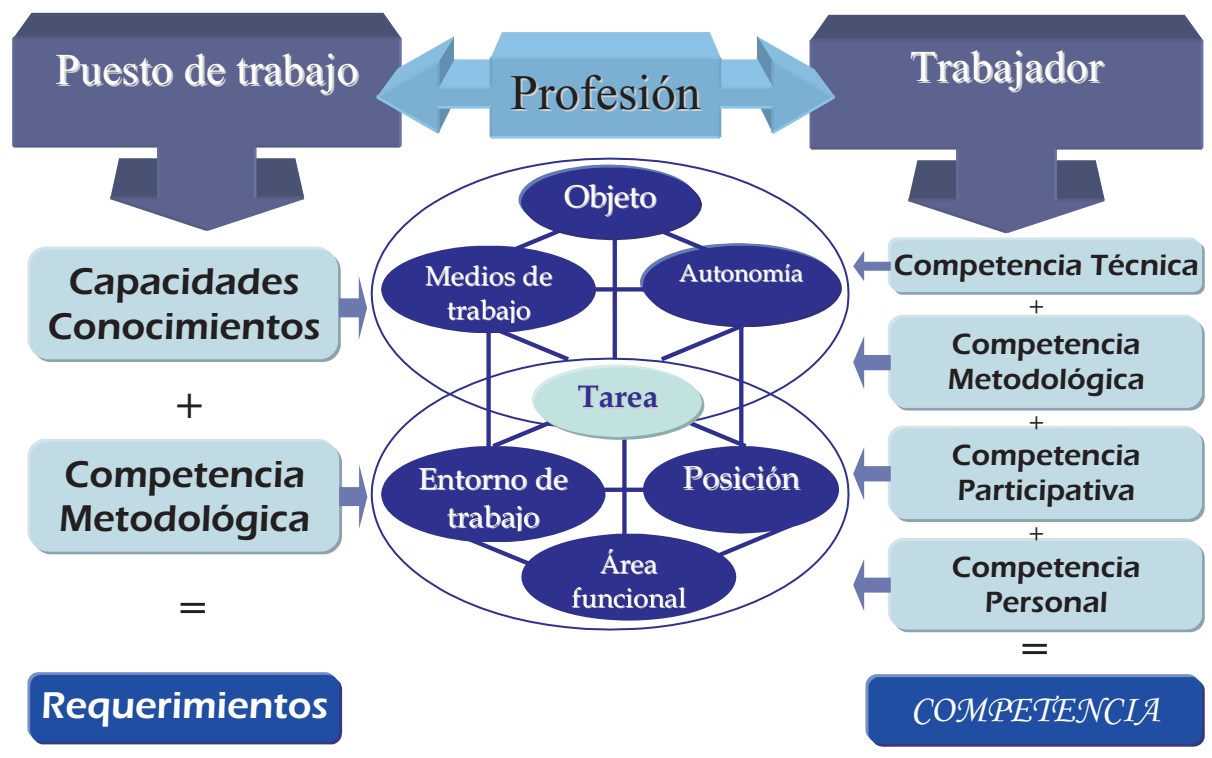

Gráfico 2. Configuración actual de la profesionalidad. (Alex, 1991, citado en Echeverría, 2001- 2008) 
Pero, ¿qué entendemos por profesionalizar la Función Tutorial Sanitaria?: entendemos que es profesionalizar la figura del tutor sanitario, es decir, definir sus competencias y cualificaciones en beneficio de su desarrollo y carrera profesional.

Según las conclusiones alcanzadas en el XVI Congreso de la Sociedad Española de Educación Médica (2003), es la expresión del desarrollo de un trabajo hecho con rigor, con la metodología e instrumentos adecuados, por los miembros de plantilla que están acreditados como tutores por un periodo determinado de tiempo. Para estos analistas, la profesionalización debe contemplar el desarrollo de los siguientes aspectos:

- Definir el perfil del tutor y los criterios para su acreditación.

- Procurar rigor en la acción tutorial (formación).

- Idear un sistema de evaluación de la acción tutorial (re- acreditación).

- Reconocer de manera efectiva la función del tutor (carrera profesional).

Parece razonable pensar que el primer paso para abordar el debate sobre la acreditaciónreacreditación del tutor sanitario es definir de forma clara y precisa cuáles es su perfil profesional y cuáles son las competencias profesionales que éstos deben reunir (Boendermaker y cols., 2000). Pero no sería justo plantear un sistema de acreditación- reacreditación sin tomar como referencia la legislación existente y los esfuerzos que se han hecho en diversas comunidades de nuestro país, por ejemplo, Cataluña, por crear sistemas capaces de producir un cambio positivo en las unidades docentes (Loayssa, Fuertes y Castillo; 2001).

En este sentido, en el artículo 12.4 del Real Decreto 183/2008, por el que se determinan y clasifican las especialidades en Ciencias de la Salud y se desarrollan determinados aspectos del sistema de formación sanitaria especializada, se establece que:

"Las Administraciones Sanitarias, a fin de mejorar sus competencias en la práctica clínica y en las metodologías docentes, favorecerán que los tutores realicen actividades de formación continuada sobre aspectos tales como los relacionados con el conocimiento y aprendizaje de métodos educativos, técnicas de comunicación, metodología de la investigación, gestión de calidad, motivación, aspectos éticos de la profesión o aspectos relacionados con los contenidos del programa formativo".
Igualmente, el artículo 11.4 señala que "las comunidades autónomas adoptarán las medidas necesarias para asegurar una adecuada dedicación de los tutores a su actividad docente, ya sea dentro o fuera de la jornada ordinaria".

Como afirma Martín (2003), el tutor es una figura clave en el proceso de aprendizaje de los especialistas en formación, actúa como referente y modelo y, por tanto, ha de reunir una serie de características que garanticen que desarrollará con eficacia sus funciones. Además de los requisitos profesionales y académicos, es preciso que el tutor tenga unas condiciones humanas de entre las que resalte su capacidad de relación y comunicación con los pacientes y su entorno, así como con los profesionales del centro. También es muy importante que tenga un grado suficiente de motivación y de disponibilidad para las tareas de la tutoría, con especial énfasis en las actividades docentes.

Para este autor, las siguientes características constituyen elementos a considerar dentro del perfil de un tutor, entre otras muchas:

- Especialista en el ámbito de tutorización.

- Profesional de plantilla de la institución.

- Con suficiente experiencia.

- Motivado por los aspectos docentes y de investigación.

- Con capacidad para las relaciones personales.

- Con buena capacidad organizativa.

- Habituado al manejo de bibliografía y con publicaciones en su haber.

Por su parte, Díaz Domínguez (2001) afirma que el tutor debe estar implicado profundamente en el proceso de aprendizaje del residente, lo que conlleva que el perfil profesional atribuido a éste sea muy exigente, destacando las siguientes características:

- Poseer gran conocimiento de la especialidad, desde el punto de vista tanto teórico como práctico.

- Ofrecer una imagen de coherencia y respeto, ya que debe procurar ser un modelo de comportamiento tanto para los residentes como para el resto de compañeros.

- Ser crítico y capaz de señalar las contradicciones que se susciten en la práctica diaria.

- Ser innovador y creativo, estimulando la solución de los problemas clínicos que se planteen.

- Ser reflexivo y autocrítico, replanteándose formas de orientar los problemas que surgen en el quehacer diario. 
- Mostrar una actitud positiva que se traduzca en un comportamiento dinámico y proactivo.

- Estar comprometido y motivado con la labor que realiza para transmitirlo al residente.

- Procurar el cumplimiento del programa formativo y los objetivos establecidos.

- Conocer y saber aplicar metodologías docentes y de evaluación, de gran importancia en el proceso de aprendizaje continuo del residente.

- Ser un buen comunicador.

- Manejar técnicas de trabajo en grupo y saber trabajar en equipo.

- Conocer a los residentes con los que debe establecer una relación de confianza y respeto.

Este autor concluye con la afirmación de que actualmente el tutor debe ser un experto en la gestión de competencias.

Sobre el perfil profesional del tutor se especifica en el artículo 11.1 del Real Decreto $183 / 2008$ que "...se adecuará al perfil profesional diseñado por el programa formativo de la correspondiente especialidad". Consideramos que este perfil profesional se encuentra más relacionado con la faceta de profesional sanitario especialista que con el propósito principal, funciones y competencias atribuidas a un tutor para llevar a cabo su cometido.

Bajo nuestro punto de vista, establecer el perfil profesional del tutor sanitario es el primer paso que debe darse en el camino hacia su profesionalización. En él quedará ampliamente reflejado cuáles son las funciones y tareas asociadas a la acción tutorial, así como las competencias necesarias para desempeñarla con éxito.

Entendemos que el perfil profesional es la descripción minuciosa de todas las tareas, deberes, riesgos, obligaciones y responsabilidades que conlleva la función tutorial, así como los requisitos exigidos al tutor que pretende llevarla a cabo.

Barbier (1997:87) lo define como el "subconjunto de capacidades específicamente producidas en el ámbito de las experiencias profesionales o de las trayectorias profesionales". La elaboración del perfil profesional requiere realizar análisis exhaustivos y sistemáticos que describan y descompongan, por una parte, las funciones, tareas, operaciones, condiciones de trabajo y otros aspectos que en conjunto conforman un puesto de trabajo (profesiografía) $y$, por otra, que identifiquen las capacidades físico-intelectuales necesarias, la experiencia, la naturaleza, el grado de responsabilidad, el medio ambiente fisiológico, medio ambiente social, condiciones de remuneración y condiciones de promoción (profesiograma). Se trata de dos campos conceptualmente distintos, el de las actividades laborales y el que se refiere a las aptitudes y características humanas.

Es comúnmente aceptado, que el perfil profesional es el conjunto de funciones, roles, conocimientos, habilidades y destrezas, actitudes y valores que un recurso humano determinado pone en práctica para el desempeño de una profesión.

Con este trabajo pretendemos contribuir al esfuerzo realizado hasta el momento por clarificar la figura del tutor sanitario y contribuir a su profesionalización, intentando describir las funciones que rigen la acción tutorial en la formación de especialistas sanitarios, paso previo e ineludible si se pretenden establecer sus competencias, su perfil profesional o iniciar procesos de formación y/o acreditación.

\section{NUEVOS RETOS DEL PROFESIONAL SANITARIO EN EL SIGLO XXI}

Los cambios que están teniendo lugar continuamente en nuestra sociedad plantean desafíos y nuevas tendencias socio- laborales a las que es preciso responder. Como ya comentamos en el punto anterior, la gran transformación estructural de nuestros días trae como consecuencia cambios en las lógicas de producción y en las organizaciones, lo que a su vez provoca que el mercado laboral demande a los profesionales del siglo XXI una serie de competencias no contempladas hasta el momento.

En este sentido algunas de las competencias que se les atribuye a los profesionales sanitarios de hoy en día son las siguientes: una formación integral comprometida con la recuperación, cuidado y promoción de la salud, una concepción integral del hombre y la salud, una sólida formación científica, estar preparado para liderar y trabajar en equipos multidisciplinares, poseer habilidades directivas y de gestión, etc. (Risco, 2007). En este caso, corresponde a la formación de pregrado, postgrado y, en general, a la formación continua, llevada a cabo en el Sistema Nacional de Salud, responder a las exigencias y desafíos provenientes del contexto laboral, para dar lugar a profesionales sanitarios válidos en el desarrollo de sus funciones. 
Será preciso, por tanto, introducir cambios en los sistemas de enseñanza/aprendizaje que ayuden a los futuros profesionales a adquirir realmente las competencias que demanda el mercado laboral, puesto que una enseñanza centrada en la transmisión del conocimiento, por citar una característica de la formación tradicional, poco o nada propiciará la adquisición por parte de los estudiantes/aprendices de habilidades de comunicación, por ejemplo.

Si se producen cambios en los requerimientos profesionales, es evidente que también deben producirse cambios en la formación que reciban los futuros profesionales. De esta manera, se modificarán los contenidos de la formación, la metodología docente, la forma de evaluar el aprendizaje... por lo que evolucionará también el perfil profesional del formador, en nuestro caso, del tutor, porque se han visto alteradas sus funciones. Su misión ya no es transmitir en voz alta únicamente una serie de conocimientos en su consulta, o por el pasillo, suponiendo que el residente se encuentra a su lado realizando una escucha activa. Como hemos dicho con anterioridad, su misión es la de garantizar y formar profesionales de manera integral, comprometidos con la recuperación, cuidado y promoción de la salud, con una concepción integral del hombre y la salud, con una sólida formación científica, preparados para liderar y trabajar en equipos multidisciplinares y poseer habilidades directivas y de gestión, entre otras competencias.

\section{EVOLUCIÓNDELPERFILDELPROFESIONAL DE LA SALUD}

A través de la historia, el profesional sanitario, y fundamentalmente el médico, ha sido el profesional más respetado por la sociedad, por ser poseedor de conocimientos altamente especializados, de difícil acceso al ciudadano común, y por tener la vocación de ponerlos al servicio de la sociedad en general y acorde con un estricto código de ética profesional (Risco, 2007).

Debido a los cambios estructurales y sociales que hemos ido viendo, la relación de confianza entre el profesional sanitario y la sociedad se ha visto profundamente perturbada, a partir de las dos últimas décadas del siglo $X X$. Se puede constatar como se ha generado insatisfacción en los pacientes por la falta de relación entre coste y calidad en los servi- cios de atención a la salud; en consecuencia, han aparecido nuevas formas de financiación de la misma, y las dificultades de acceso a los servicios y la poca relación humana que se da con los distintos profesionales sanitarios no deja de ser un problema (Risco, 2007). Los profesionales sanitarios, por su parte, muestran frustración a la hora de desempeñar su labor y brindar el mejor servicio a los pacientes debido a restricciones en su tiempo personal, consecuencia de amplias jornadas de trabajo, escasez de incentivos o pérdida de control sobre sus decisiones clínicas (Cruess, Cruess y Johnston, 2000).

A la vista de esta situación, es preciso reconstruir la relación de confianza entre el profesional sanitario y la sociedad. El profesional sanitario debe aprender a desempeñarse en este nuevo contexto, manteniendo las características más importantes que han definido su profesionalismo: vocación y moralidad (Risco, 2007). En este contexto, les corresponde a las instituciones de educación superior en Ciencias de la Salud (Facultades de Medicina, Escuelas de Enfermería...) un rol muy importante en la formación del nuevo tipo de profesional sanitario que la sociedad demanda; por ello, revisamos a continuación algunos de los principales desafíos que enfrenta la sociedad actual, los que deben ser tomados en cuenta en el diseño de un nuevo currículo.

En primer lugar, nos encontramos con el desarrollo científico y tecnológico. Como ya hemos comentado, vivimos en la sociedad de la información y del conocimiento en la que el saber se renueva a una velocidad vertiginosa. Además, el desarrollo de las TICs permite la difusión de grandes volúmenes de información a través de redes digitales en tiempo real y a un coste cada vez menor. Por tanto, actualmente el objetivo de la educación no debe limitarse a la transmisión de información, sino, más bien, a lograr que los estudiantes aprendan a seleccionarla, evaluarla, interpretarla, clasificarla y utilizarla, es decir a gestionar la información y su propio conocimiento (Martínez Clares, 2002). Para lograr este objetivo, la educación debe desarrollar en los estudiantes su capacidad creativa, de reflexión, de resolución de problemas... Es necesario orientar la educación de pregrado hacia una formación más integral y motivar al estudiante para continuar con este mismo tipo de formación en las etapas de especialización y postgrado, ade- 
más del permanente aprendizaje autónomo que deberá desarrollar a lo largo de su vida.

Otro de los desafíos a los que se enfrenta la sociedad actual es el de la globalización, "un fenómeno que comprende el movimiento transnacional de bienes y servicios, así como de personas, inversiones, ideas, valores y técnicas, y cuya acelerada evolución está sustentada en la utilización cada vez más intensa del conocimiento y la tecnología" (Risco, 2007: 5). Gracias a este fenómeno se hace posible cada vez con mayor fuerza la movilidad de profesionales sanitarios y, por tanto, la interacción de estos profesionales sanitarios de distinta procedencia en un mismo centro de trabajo. Éste es precisamente una de los fines primordiales del Espacio Europeo de Educación Superior, para lo que se hace necesario establecer un conjunto de competencias esenciales que caractericen a dichos profesionales independientemente del lugar donde se hayan formado. Por lo tanto, "el reto actual en la educación superior, es formar profesionales sanitarios capaces de desempeñarse en un entorno global sin descuidar las necesidades locales de salud de la población" (Risco, 2007: 5).

De la misma manera que se hace necesario establecer una serie de competencias profesionales esenciales, se hace también indispensable la implantación de mecanismos de evaluación y acreditación de los profesionales sanitarios en ejercicio, para garantizar internacionalmente su calidad. Por ello, la World Federation for Medical Education (WFME, 2003) ha elaborado un conjunto de Estándares Internacionales de Calidad en Educación Médica con el propósito de brindar una referencia tanto a las instituciones de educación superior en Ciencias de la Salud como a los organismos que acreditan la educación médica de postgrado y continua en el mundo. De esta forma, el profesional sanitario egresado de una facultad o escuela, de un centro sanitario acreditado o que se actualiza a través de la formación continua, tendrá una credencial de calidad reconocida internacionalmente.

Si continuamos con los desafíos con los que se encuentra el profesional sanitario actual, nos encontramos con que la práctica sanitaria y las condiciones de su ejercicio han sufrido profundos cambios debido a las transformaciones sociales ya comentadas, a los costes de la atención a la salud en relación a su calidad y a las nuevas formas de financiarla, entre otras razones.

Se ha pasado de un estilo de práctica individual, en la consulta particular, al ejercicio grupal en asociación con otros profesionales sanitarios de distintas ramas de la salud. Este hecho exige al profesional sanitario la capacidad de trabajar en equipos multidisciplinares, además de competencia en gestión y liderazgo.

Los avances científicos y tecnológicos, a su vez, están propiciando una mayor calidad en la atención de forma ambulatoria, lo que trae como consecuencia un menor coste y una mayor rapidez en la recuperación del paciente, olvidando el ingreso del paciente por largos periodos de tiempo en el hospital, en el caso de algunas enfermedades.

En la línea anterior, hoy día existe la posibilidad de diagnosticar, prevenir y curar enfermedades que antes eran irremediables gracias a los avances científicos y tecnológicos.

Esto ha aumentado la esperanza de vida de la población y ha incrementado el número de pacientes de edad avanzada y de pacientes crónicos que acuden a los servicios de salud requiriendo nuevas competencias por parte de los profesionales de la Salud.

Señalar también que la diferencia en el coste y la calidad de la atención recibida en los servicios sanitarios ha impulsado el desarrollo de diversas formas de financiación de la misma. Esto hace necesario y conveniente la creación de sistemas de auditoria, con el fin de verificar el buen funcionamiento de los centros sanitarios y la calidad de sus profesionales.

Por último, mencionar que las expectativas de los pacientes también han cambiado. El acceso a la información ya no es un problema, por lo que los pacientes reclaman la mejor atención posible y desean estar informados y ser partícipes de las decisiones respecto a su salud.

El profesional sanitario debe ser capaz de comunicarse de forma efectiva y empática con el paciente, informándolo adecuadamente.

Además, los pacientes esperan de estos profesionales un trato humano y cercano, reclamando de ellos responsabilidad legal y ética sobre el cuidado de su salud, por lo que actualmente las Ciencias de la Salud deben incluir la comunicación como una de las competencias esenciales en sus perfiles profesionales. 


\section{NUEVAS TENDENCIAS EN EDUCACIÓN SANITARIA}

Ante todos estos desafíos y requerimientos nos preguntamos, ¿cuáles deberían ser las nuevas tendencias en la educación sanitaria para el siglo $\mathrm{XXI}$ ?

Contestaremos haciendo alusión a las competencias mínimas que tendrá que tener el profesional sanitario del siglo XXI y a la necesidad de establecer un nuevo currículo formativo, a partir de un nuevo enfoque de formación basado en competencias que utilice como metodologías más apropiadas para la adquisición de las mismas el Aprendizaje basado en Problemas y el Aprendizaje de Habilidades Clínicas Básicas.

De esta forma, el profesional sanitario del siglo XXI debería tener las siguientes características y competencias (adaptación de Risco, 2007):

- Adquirir una formación integral, comprometida con el cuidado, la recuperación y promoción de la salud, así como con la prevención de las enfermedades.

- Poseer una concepción integral del hombre y la salud, que incluye aspectos biológicos, psicológicos, socioculturales y ambientales.

- Tener una sólida formación científica que le permita ser competente para evaluar, investigar, diagnosticar y resolver los problemas de salud del individuo y la población de manera efectiva e innovadora, teniendo una perspectiva integral en el análisis de las mismas.

- Estar orientado a la calidad del servicio y a la atención del usuario con una actitud humana y ética.

- Estar preparado para liderar y trabajar en equipos multidisciplinares.

- Conocer la organización en la que desarrolla su labor profesional.

- Poseer habilidades directivas y de gestión.

- Tener la motivación y la capacidad suficientes para seguir aprendiendo de forma autónoma durante toda la vida.

Igualmente, el nuevo currículo de estos profesionales tendrá que tener las siguientes características (adaptación de Risco, 2007):

- Diseñarlo de acuerdo a un modelo educativo basado en competencias y centrado en el estudiante, a diferencia del modelo tradicional, basado en la mera transmisión de contenidos y centrado en el profesor.
- Plantearlo de manera que propicie una formación integral del estudiante, es decir, que incluya competencias personales y profesionales. - Reducir la cantidad de información, seleccionar los contenidos relevantes, integrar vertical y horizontalmente los mismos e introducir las ciencias básicas junto con la clínica.

- Emplear métodos de enseñanza que favorezcan la participación activa del estudiante en el proceso de enseñanza - aprendizaje.

- Procurar un desarrollo temprano de las habilidades clínicas y comunicativas con el paciente y sus familiares, equilibrando las experiencias curativas y preventivas, así como las experiencias comunitarias y hospitalarias.

Del mismo modo, corroboramos que el enfoque de Formación basada en Competencias (FBC), más conocida en la literatura internacional como Outcome Based Education, es considerado uno de los cambios más importantes en la educación para el siglo XXI. Este es el enfoque de formación predominante en las instituciones de educación superior de Ciencias de la Salud y el que recomiendan los principales organismos rectores y evaluadores de la Educación Sanitaria.

A lo largo de este artículo hemos podido conocer y valorar el nuevo profesional sanitario que reclama la sociedad actual y que deberá ser formado por las instancias de educación superior pertinentes. Una vez finalizada esta formación de pregrado, llega el periodo de formación para la obtención del título de especialista. Esta formación, conocida popularmente como sistema de residencia, se basa en completar y enriquecer las competencias adquiridas durante la carrera y se caracteriza por la supervisión directa de un colega con mayor experiencia. Este colega se convertirá en la persona que debe facilitarle la adaptación a este nuevo perfil profesional que reclama tanto la sociedad como los centros del sistema sanitario, con el fin de dar una mejor respuesta a las necesidades de sus usuarios y clientes. Pero, este supervisor, guía, orientador, facilitador..., en definitiva, el tutor, ¿responde a ese perfil? ¿Ha sabido adaptarse al cambio producido en la configuración de su profesionalidad? ¿Reúne las características básicas atribuidas a su figura? ¿Es capaz de cumplir de manera adecuada con la amalgama de funciones que se le atribuyen?

En nuestro país, el Programa Formativo de la Especialidad de Medicina Familiar y Comu- 
nitaria (2005) fue el primer programa en el que se contempló la figura del tutor y se introdujeron conceptos como el de competencia y perfil profesional. Detallamos a continuación lo que de él se específica, generalizándolo al resto de especialidades:

"El Tutor es aquel profesional sanitario, en ejercicio activo, al que se le reconoce un nivel formativo y un entorno laboral adecuado que permite acreditarlo como capacitado para favorecer el aprendizaje de los conocimientos, habilidades y actitudes propios de una formación (...) especializada y que asume colaborar de forma activa en el proceso de enseñanzaaprendizaje de los especialistas en formación, dentro de un contexto institucionalizado.

El tutor es una figura esencial en la formación del residente y especialmente en el contexto de (...) cualquier programa que entre sus características definitorias tiene la flexibilidad, lo que implica la individualización y personalización del programa formativo adaptando contenidos, tiempos y métodos a las necesidades propias de cada residente.

El tutor debe fundamentalmente reunir dos características:

- Ser un buen (...) profesional sanitario: su perfil competencial debe adecuarse al descrito en (...) cada programa formativo, dependiendo de su especialidad.

- Ser un buen docente, ya que según sea su actitud hacia la enseñanza, su preparación específica, su capacidad para transmitir conocimientos o su manejo de las relaciones interpersonales, el residente obtendrá una mejor o peor preparación".

La primera característica puede estar garantizada en el tutor por la formación recibida (pregrado, postgrado y formación continua) y la experiencia diaria en su lugar de trabajo, pero ¿y la segunda característica?, ¿le viene dada por sus años de formación?, ¿la adquiere a través de su experiencia laboral? Bajo nuestro punto de vista, ni a partir de la una ni de la otra. Un profesional sanitario que decida hoy en día llevar a cabo la función tutorial dentro del sistema nacional de salud no está suficientemente preparado para desarrollarla de manera óptima ya que, independientemente de que no se encuentren establecidas de manera clara sus funciones y por lo tanto la acción tutorial sea considerada como algo abstracto e inespecífico, no se encuentra formado para ello. No se dispone de planes de formación inicial que capaciten a los tutores para su acreditación y mucho menos de planes de formación continua que aseguren el desarrollo profesional del tutor sanitario.

Entonces,... ¿Cómo puede responder el tutor de especialistas a la formación del profesional sanitario del siglo XXI?

El tutor podrá proporcionar una formación adecuada al nuevo profesional sanitario del siglo XXI cuando se encuentre especificado su perfil profesional, cuando reciba una formación cualificada y cuando sus funciones y competencias se encuentren claramente identificadas y concretadas.

\section{REFERENCIAS}

Alex, L. (1991). Descripción y registro de las cualificaciones. El concepto de cualificación. Formación Profesional, 2:23-37.

Barbier (1997). L'approche transversale. Paris: Anthropos. Boendermaker, P. M. y colb. (2000). What are the characteristics of the competent general practitioner trainer? Family Practice; 17: 547- 553.

Bunk, G. P. (1994). La transmisión de las competencias en la formación y perfeccionamiento de profesionales de la RFA. Revista Europea de Formación Profesional; 1: 8-14.

Canadian Medical Education Directions for Specialists 2000 Project (1996). Skills for the new millennium: report of the societal needs working group. Otawa: The Royal College of Physicians and Surgeons of Canada's.

Conclusiones del XVI Congreso de la Sociedad Española de Educación Médica (2003). Revista Educación Médica; 6(4): 125- 126.

Cruess, R. L.; Cruess, S. R. y Johnston, S. E. (2000). Professionalism: an ideal to be sustained. The Lancet; 356: 156- 159.

Delcourt, J. (1991). Nuevas presiones a favor de la formación en la empresa. Revista Europea de Formación Profesional; 17: 3- 14.

Delors, J. (Coord.) (1996). La educación encierra un tesoro. Madrid: Santillana. UNESCO.

Díaz Domínguez, J. (2001). Tutoría y supervisión de la formación de especialistas sanitarios: perfil, funciones, acreditación y reconocimientos. Conclusiones. En Jornada de Comisiones Docentes y Asesoras de la Comunidad de Madrid. Situación actual y perspectivas de futuro de la formación de especialistas sanitarios en la Comunidad de Madrid (pp. 114-119). Madrid: Agencia Laín Entralgo.

Echeverría, B. (2001). Configuración Actual de la Profesionalidad. Letras de Deusto; 31 (91): 35- 55.

Echeverría, B. (2002). Gestión de la Competencia de Acción Profesional. Revista de Educación Educativa; 20 (1): 7- 43. 
Echeverría, B. (2005). Competencia de Acción de los Profesionales de la Orientación. Madrid: ESIC.

Echeverría, B. [Coord.] (2008). Orientación Profesional. Barcelona: UOC.

Fernández- Ríos, M. (1995). Análisis y descripción de puestos de trabajo. Madrid: Díaz de Santos.

Loayssa, J. R.; Fuertes, C. y Castillo, M. (2001). Acreditación y reacreditación de tutores. Tribuna Docente; Supl. 3 (2): 13-20.

Martín Zurro, A. (2003) Acreditación y reconocimiento de los tutores. Incentivación de la acción tutorial. Revista Educación Médica; 6(3): 7-8.

Martínez Clares, P. (2002). La orientación psicopedagógica: modelos y estrategias de intervención. Madrid: EOS.

Martínez Clares, P.; Rubio, M.; Garvía, C. y Martínez Juárez, M. (2003). Estudio cualitativo inicial de los Servicios de Orientación de la Universidad de Murcia. Comunicación presentada al XI Congreso Nacional de Modelos de Investigación Educativa:"Investigación y Sociedad." Granada, septiembre de 2003.
Morán Barrios, J. M. (2003). ¿Es necesaria y compatible la existencia del tutor de médicos residentes dentro de nuestras estructuras asistenciales? Revista Educación Médica; 6(3): 10- 11.

ORDEN SCO/1198/2005, de 3 de marzo, por la que se aprueba y publica el programa formativo de la especialidad de Medicina Familiar y Comunitaria. BOE de 3 de mayo de 2005.

Real Decreto 183/2008, de 8 de febrero, por el que se determinan y clasifican las especialidades en Ciencias de la Salud y se desarrollan determinados aspectos del sistema de formación sanitaria especializada. BOE de 21 de febrero de 2008.

Risco, G. (2007). Educación Médica: Nuevas Tendencias, desafíos y oportunidades. Revista de Investigación en Docencia Universitaria; 3 (1).

Taylor, F. W. (1911). Principles of scientific management. Nueva York: Hasper.

Vargas, F. y cols. (2001). El enfoque de la competencia laboral: Manual de formación. Montevideo: CINTERFOR.

World Federation Medical Education (2003). Postgraduate Medical Education. WFME Global Standards for Quality Improvement. Denmark: University of Copenhaguen. 